\title{
Forward Physics at the LHC
}

\author{
David d'Enterria
}

CERN, PH-EP, CH-1211 Geneva 23, Switzerland

\begin{abstract}
Small-angle detectors at the LHC give access to a broad physics programme within and beyond the Standard Model (SM). We review the capabilities of ALICE, ATLAS, CMS, LHCb, LHCf and TOTEM for forward physics studies in various sectors: soft and hard diffractive processes, exclusive Higgs production, low- $x$ QCD, ultra-high-energy cosmic-rays, and electro-weak measurements [1].
\end{abstract}

\section{Introduction}

The CERN Large Hadron Collider (LHC) will provide the highest energy proton-proton and ion-ion collisions in the lab to date. The multi-TeV energy of the colliding beams opens up a phase space for particle production in an unprecedented range spanning $\Delta \eta \sim 20$ units of rapidity ${ }^{\mathrm{a}}: y_{\text {beam }}=\operatorname{acos}(\sqrt{s} / 2)=9.54$ for p-p at $14 \mathrm{TeV}$. As a general feature, particle production in hadronic collisions is peaked at central rapidities $(|y| \lesssim 3$ at the LHC), whereas most of the energy is emitted at very low angles (Fig. 1 left).
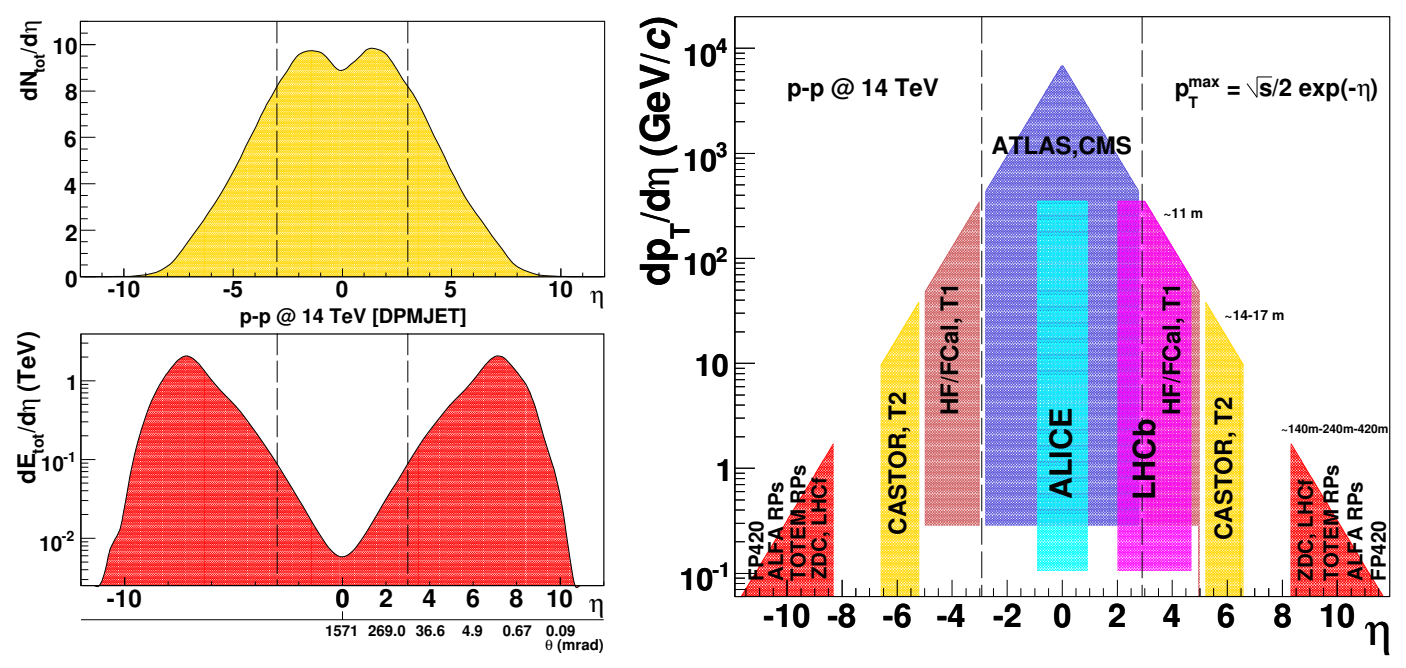

Figure 1: Left: Pseudo-rapidity distributions for the total hadron multiplicity (top) and energy (bottom) in p-p at $14 \mathrm{TeV}$ as given by the DPMJET3 model [2]. Right: Approximate $p_{T^{-}-\eta}$ coverage of current (and proposed) detectors at the LHC.

All LHC experiments feature detection capabilities at forward rapidities without parallel compared to previous colliders (Figures 1 right, and 2):

- ATLAS $[4,5]$ and CMS $[6,7]$ not only cover the largest $p_{T}-\eta$ ranges at mid-rapidity for hadrons, electrons, photons and muons, but they feature extended instrumentation

\footnotetext{
aThe rapidity can be thought of as the relativistically-invariant measure of longitudinal velocity. Often the pseudorapidity $\eta=-\ln \tan (\theta / 2)$ which depends only on the polar angle wrt the beam axis, is used instead.
} 
at distances far away from the interaction point (IP): $\pm 11 \mathrm{~m}$ (ATLAS FCal and CMS HF hadronic calorimeters), $\pm 14 \mathrm{~m}$ (CMS CASTOR sampling calorimeter [8]), $\pm 140 \mathrm{~m}$ (Zero-Degree-Calorimeters, ZDCs [5, 9]), and $\pm 240 \mathrm{~m}$ (ATLAS Roman Pots, RPs [5]).

- ALICE [10] and LHCb [11] have both forward muon spectrometers in regions, $2 \lesssim$ $\eta \lesssim 5$, not covered by ATLAS or CMS. (In addition, ALICE has also ZDCs at \pm 116 $\mathrm{m}[12])$.

- The TOTEM experiment [13], sharing IP5 with CMS, features two types of trackers (T1 and T2 telescopes) covering $3.1<|\eta|<4.7$ and $5.2<|\eta|<4.7$ respectively, plus proton-taggers (Roman Pots) at \pm 147 and $\pm 220 \mathrm{~m}$.

- The LHCf [14] tungsten-scintillator/silicon calorimeters share the location with the ATLAS ZDCs $\pm 140 \mathrm{~m}$ away from IP1.

- The FP420 R\&D collaboration $[15,16]$ aims at installing proton taggers at $\pm 420 \mathrm{~m}$ from both ATLAS and CMS IPs.

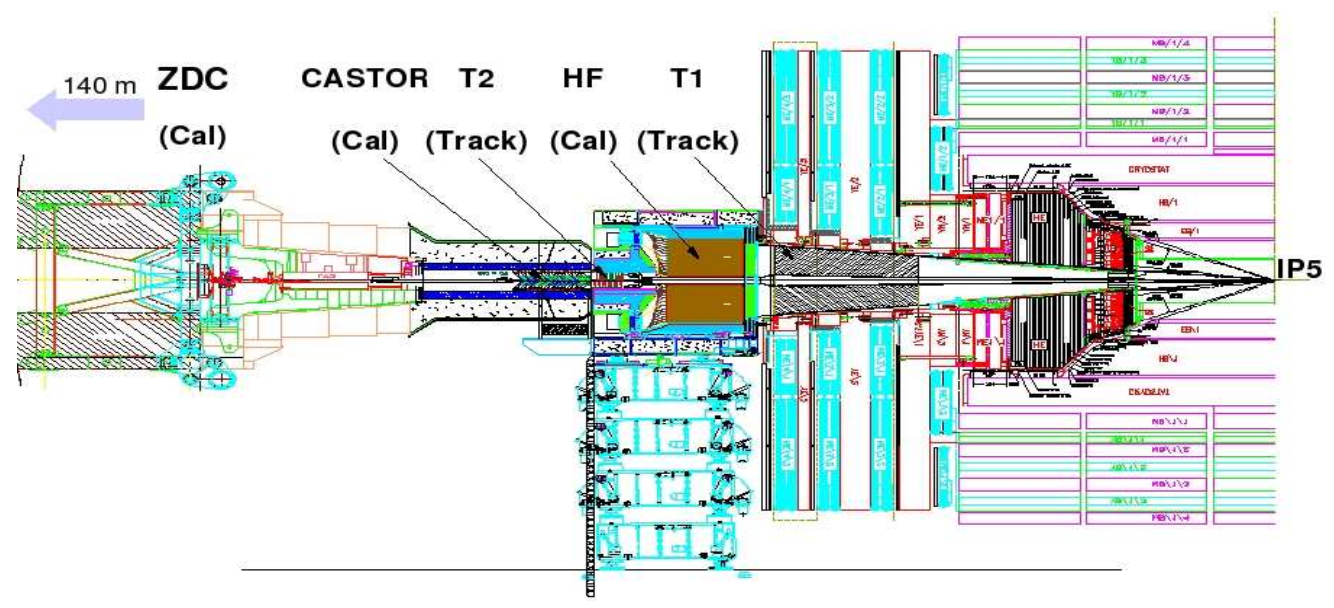

Figure 2: Layout of the detectors in the CMS/TOTEM forward region [3].

Near beam instrumentation provides access to a rich variety of physics measurements when used in three possible modes: (i) as detectors to directly measure a given final-state produced in the reaction (e.g. a jet in CASTOR, or a photon in LHCf/ZDC), (ii) as tagging devices for the (diffractively or elastically) scattered protons (in Roman Pots or other p-taggers), and/or (iii) as vetoing devices of final-state particles produced in the collision (e.g. requiring no hadronic activity within a large rapidity range).

The following forward physics topics will be discussed in this short review:

1. Diffraction (soft and hard) and elastic scattering $[17,18]$. Measurements like the total p-p cross-section, the rapidity-gap survival probability, and hard diffraction crosssections (heavy-Q, dijets, vector-bosons, ...) are accessible with the TOTEM and ALFA Roman Pots and/or by requiring a large enough rapidity gap in one (or both) of the forward hemispheres (e.g. HF+CASTOR in CMS). 
2. Central exclusive production of the Higgs boson and other heavy (new) particles $[19,20]$ can be studied combining the FP420 proton-taggers with the central ATLAS and CMS detectors.

3. The phenomenology of low- $x$ QCD - parton saturation, non-linear QCD evolution, small- $x$ PDFs, multi-parton scattering [21] - can be studied via the measurement of hard QCD cross-sections in the forward direction (e.g. jets, direct- $\gamma$ in HF/FCal, CASTOR,...$)$ or in exclusive photoproduction processes $(\gamma-\mathrm{p}, \gamma$-A interactions $)$ tagged with forward protons (neutrons) in RPs (ZDCs).

4. Models of hadronic interactions of ultra-high-energy (UHE) cosmic-rays in the upper atmosphere [22] can be effectively tuned by measuring in CASTOR, TOTEM, LHCf and ZDCs, the energy $(d E / d \eta)$ and particle $(d N / d \eta)$ flows in p-p, p-A, and A-A collisions.

5. Electroweak interactions: Ultrarelativistic protons and ions generate fluxes of (equivalent) photons which can be used for a rich programme of photoproduction studies at $\mathrm{TeV}$ energies [23]. Photon-induced interactions, tagged with forward protons (neutrons) in the RPs (ZDCs), allow one e.g. to measure the beam luminosity (via the pure QED process $\gamma \gamma \rightarrow l^{+} l^{-}$) or to study (anomalous) gauge boson couplings (via $\gamma$-p, $\gamma$-A $\rightarrow$ p n $W$, or $\gamma \gamma \rightarrow Z Z, W W)$.

\section{Total and elastic cross sections}

The measurement at the LHC of the total p-p cross-section and $\rho$-parameter (ratio of real to imaginary part of the forward elastic scattering amplitude) provides a valuable test of fundamental quantum mechanics relations [24] like the Froissart bound $\sigma_{\text {tot }}<$ Const $\ln ^{2} s$, the optical theorem $\sigma_{\text {tot }} \sim \operatorname{Im} f_{\text {el }}(t=0)$, and dispersion relations $\operatorname{Re} f_{\text {el }}(t=0) \sim \operatorname{Im} f_{\text {el }}(t=0)$.
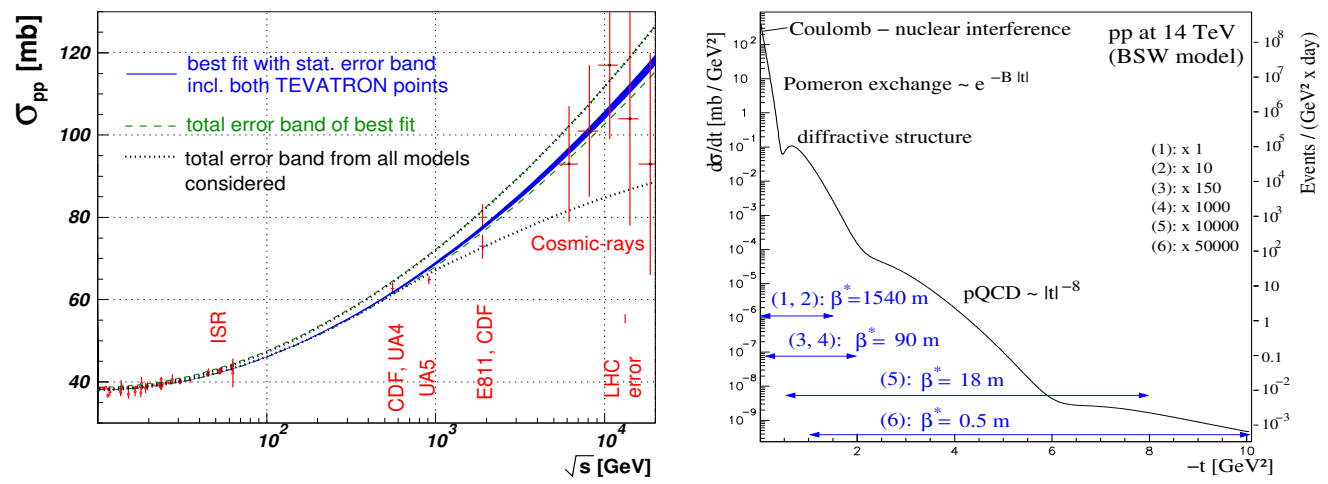

Figure 3: Left: COMPETE predictions [25] for $\sigma_{\text {tot }}$ with statistical (blue solid) and total (dashed) errors (including the Tevatron ambiguity) compared to existing data. Right: Prediction for elastic p-p scattering at the LHC with various beam optics settings [26].

The main goal of TOTEM is to obtain a precise $(\sim 1 \%)$ measurement of the total and elastic p-p cross-section over a large range of (low) 4-momentum transfers $-t \approx p^{2} \theta^{2}$ (Fig. 3 ). The COMPETE [25] extrapolation values of $\sigma_{\text {tot }}=111.5 \mathrm{mb}$ and $\rho=0.1361$ at LHC are uncertain 
to within ${ }_{-8 \%}^{+5 \%}$ due to a $2.6 \sigma$ disagreement between the E710 and CDF measurements at Tevatron. In addition, TOTEM can also provide (via the optical theorem) the absolute p-p beam luminosity with reduced uncertainties using a low- $\beta$ setting.

\section{Diffractive physics}

Diffractive physics covers the class of interactions that contain large rapidity gaps (LRGs, $\Delta \eta \gtrsim 4)$ without hadronic production. Such event topologies imply colorless exchange, requiring two or more gluons in a color-singlet state (a Pomeron, $\mathbb{P}$ ). Depending on the number and relative separation of the LRGs, one further differentiates between single, double, or double-Pomeron-exchange (DPE) processes (Fig. 4).

a)

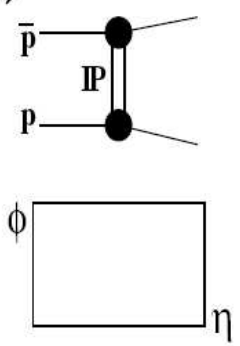

Elastic b)
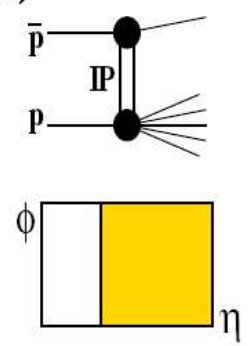

Single-Diffractive

(SD) c)
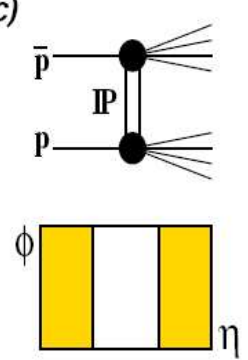

Double-Diffractive

(DD) d)
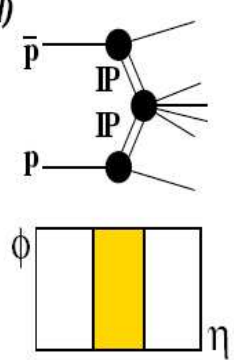

Double Pomeron Exchange (DPE)

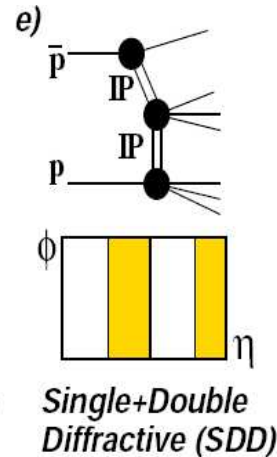

Figure 4: Event topologies in $\eta$ vs azimuth $\phi$ for elastic and diffractive p-p interactions. Shaded (empty) areas represent particle production (rapidity gaps) regions [27].

On the one hand, soft diffraction processes are controlled by non-perturbative (Regge) dynamics and constitute a significant fraction $(\sim 20 \%)$ of the total inelastic p-p cross-section. Their characterization is thus important in order to determine the pile-up backgrounds at high luminosities. On the other, hard diffraction processes involve the production of a highmass or large- $p_{T}$ state $(\mathrm{X}=\mathrm{Q} \overline{\mathrm{Q}}$, jets, $W, Z \ldots)$ and are in principle calculable perturbatively by means of the factorisation theorem and diffractive (or generalised) Parton Distribution Functions, dPDFs (GPDs). The apparent breakdown of pQCD factorization in hard diffractive processes - supported by a reduced gap-survival probability in Tevatron $\mathrm{p}-\overline{\mathrm{p}}$ compared to e-p at HERA - has important phenomenological implications for LHC [17, 18]. Of particular interest are hard exclusive DPE processes where the centrally produced system can be a new heavy particle (see Fig. 5 and next Section).

\section{Higgs (and new) physics}

Central exclusive processes (CEP, Fig. 5 left) are defined as $\mathrm{pp} \rightarrow \mathrm{p} \oplus \mathrm{X} \oplus \mathrm{p}$ where $\mathrm{X}$ is a fully measured simple state such as $\chi_{c, b}$, jet-jet $(j j), l^{+} l^{-}, \gamma \gamma, \mathrm{H}, W^{+} W^{-}, \ldots$ and ' $\oplus^{\prime}$ represents a large rap-gap $(\Delta \eta \gtrsim 4)$. Central exclusive Higgs production has recently attracted lots of experimental and theoretical attention $[16,20]$. First, the expected SM cross-sections are of order (3-10) fb (Fig. 5, right), but depending on $\tan \beta$ can be a factor of 10 (100) larger in minimal supersymmetric extensions of the SM (MSSM). Second, precise measurements of 
the proton momenta $\left(d p / p \approx 10^{-4}\right)$ allow one to measure the Higgs mass with $\sigma\left(m_{H}\right) \approx 2$ $\mathrm{GeV}$, independent of the (central) decay mode (e.g. b $\bar{b}, W W, Z Z)$. Third, spin selection rules suppress a large fraction of QCD production resulting in a very favourable 1:1 signalto-background. Fourth, due to $\mathrm{CEP} \mathrm{J}^{\mathrm{PC}}=0^{++}$selection rules, azimuthal correlations of the outgoing protons are likely to provide the only method at hand at the LHC to easily determine the Higgs quantum numbers. Given the currently preferred range of Higgs masses, $m_{H}<200 \mathrm{GeV}$, the optimal proton tagging acceptance is however beyond the current reach of TOTEM or ALFA RPs. The FP420 R\&D collaboration proposes novel technologies (moving beam-pipe, fast 10-ps Cerenkov detectors, ...) as ATLAS and CMS upgrades for proton tagging at $\pm 420 \mathrm{~m}$.
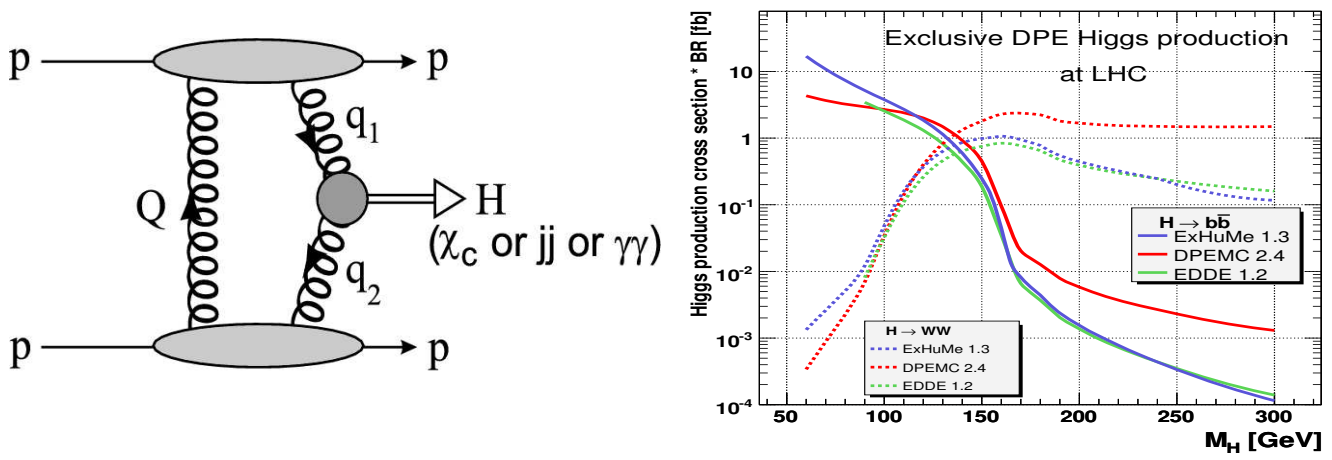

Figure 5: Left: Central exclusive Higgs production via two-gluon exchange. Right: Crosssections for the SM Higgs (b $\overline{\mathrm{b}}, W W$ channels) from various perturbative calculations [3].

\section{Low- $x$ QCD physics}

One of the main HERA observations is that the proton structure function is almost purely gluonic for values of the fractional momenta $x=p_{\text {parton }} / p_{\text {proton }} \lesssim 0.01$. Fig. 6 summarises our current knowledge of the gluon density $x G\left(x, Q^{2}\right)$ in the proton. In DIS, the main source of information so far on $x G\left(x, Q^{2}\right)$ is (indirectly) obtained from the slope of the $F_{2}$ scaling violations. Additional constraints can be obtained from $F_{2}^{\text {charm }}$ [37], and diffractive photoproduction of heavy vector mesons $(J / \psi, \Upsilon)[28]$. The most direct access will come, however, from the longitudinal structure function $F_{L}$ whose measurement has driven the last (lower energy) runs at HERA [29]. In hadron-hadron collisions, $x G$ enters directly at LO in processes with prompt photons, jets, and heavy-quarks in the final state. Below $x \approx 10^{-4}$ $\left(10^{-2}\right)$ the gluon density in the proton (nucleus) is however poorly constrained as can be seen from the right plot of Fig. 6 (Fig. 7). This is a small- $x$ regime where one expects non-linear gluon-gluon fusion effects - not accounted for in the standard DGLAP/BFKL evolution equations - to become important and tame the rise of the parton densities [31] (Fig. 7 left). Such saturation effects are amplified in nuclear targets thanks to their increased transverse parton density.

Forward instrumentation provides an important lever arm for the measurement of the low$x$ structure and evolution of the parton densities. Indeed, in a $2 \rightarrow 2$ parton scattering 

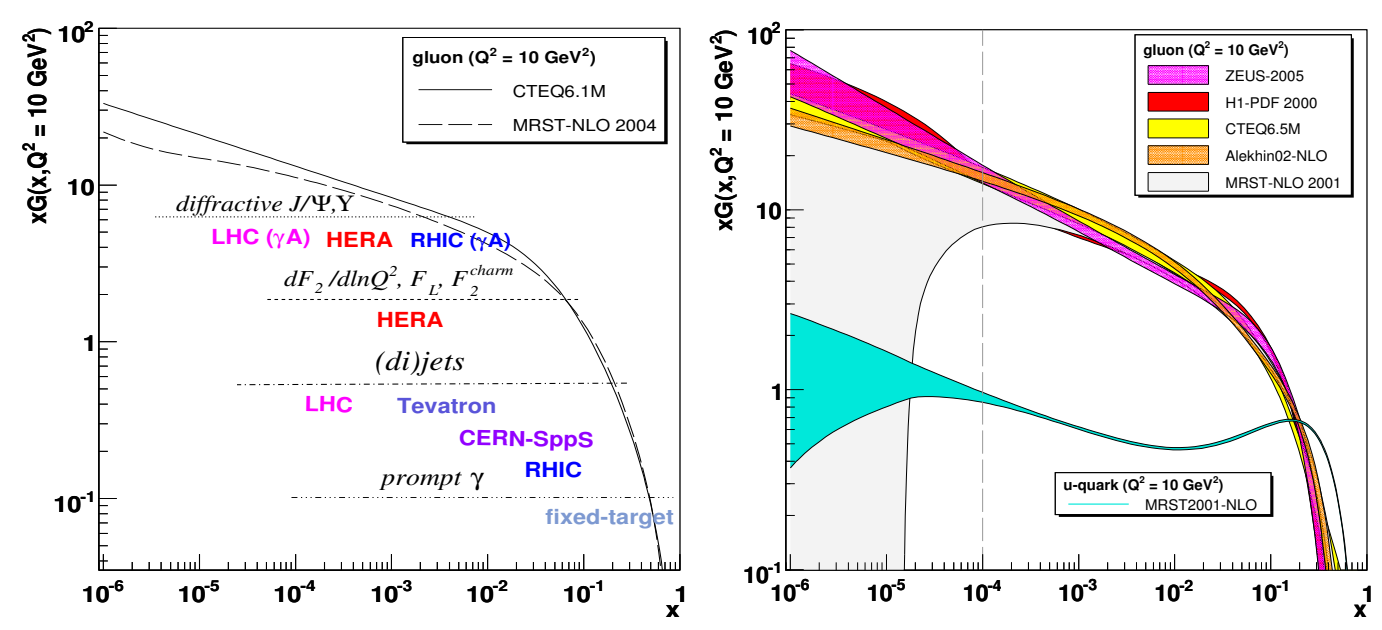

Figure 6: Left: Experimental measurements of the gluon PDF. Right: Comparison of various fits [32] of the proton $x G\left(x, Q^{2}=10 \mathrm{GeV}^{2}\right)$ (the $u$ quark PDF is also shown, for reference).

the minimum momentum fraction probed when a particle of momentum $p_{T}$ is produced at pseudo-rapidity $\eta$ is

$$
x_{\text {min }}=\frac{x_{T} e^{-\eta}}{2-x_{T} e^{\eta}} \quad \text { where } \quad x_{T}=2 p_{T} / \sqrt{s},
$$

i.e. $x_{\min }$ decreases by a factor of $\sim 10$ every 2 units of rapidity. Four representative measurements of the low- $x$ PDFs at the LHC are discussed next [21].
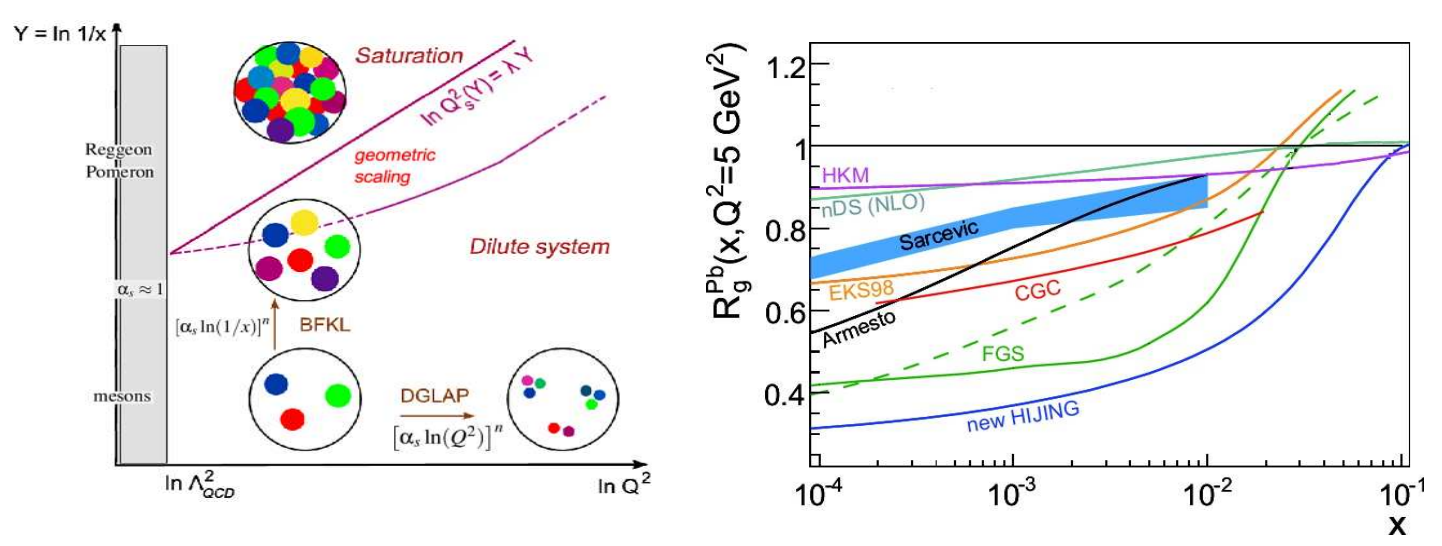

Figure 7: Left: $\mathrm{QCD} \log (1 / x)-\mathrm{Q}^{2}$ plane with the different parton evolution regimes (DGLAP, BFKL, saturation). Right: Ratios of the Pb-over-proton gluon densities versus $x$ at fixed $Q^{2}=5 \mathrm{GeV}^{2}$ from various nuclear shadowing parametrizations [30]. 
- Case study I: Forward (di)jets

The measurement of (relatively soft) jets with $p_{T} \approx 20-100 \mathrm{GeV} / c$ in p-p at $14 \mathrm{TeV}$ in the CASTOR forward calorimeter $(5.2<|\eta|<6.6)$ allows one to probe the PDFs at $x$ values as low as $x \approx 10^{-6}$ (see Fig. 8 left, for jets in ATLAS FCal and CMS HF calorimeters). In addition to the single inclusive cross-sections, the production of events with two similar transverse-momentum jets emitted in each one of the forward/backward directions, the socalled "Müller-Navelet jets" (Fig. 8 right), is a particularly sensitive measure of BFKL [33] as well as non-linear [34] parton evolutions. The large rapidity interval between the jets (e.g. $\Delta \eta \approx 10$ in the extremes of HF+ and HF-) enhances large logarithms of the type $\Delta \eta \sim \log \left(s / k_{1} k_{2}\right)$ which can be appropriately resummed using the BFKL equation. The phenomenological consequences expected in the BFKL regime are enhanced Müller-Navelet dijet rates and wider azimuthal decorrelations for increasing $\Delta \eta$ separations [35, 36]. Preliminary CMS analyses [3] indicate that such studies are well feasible measuring jets in each one of the hadronic forward (HF) calorimeters.
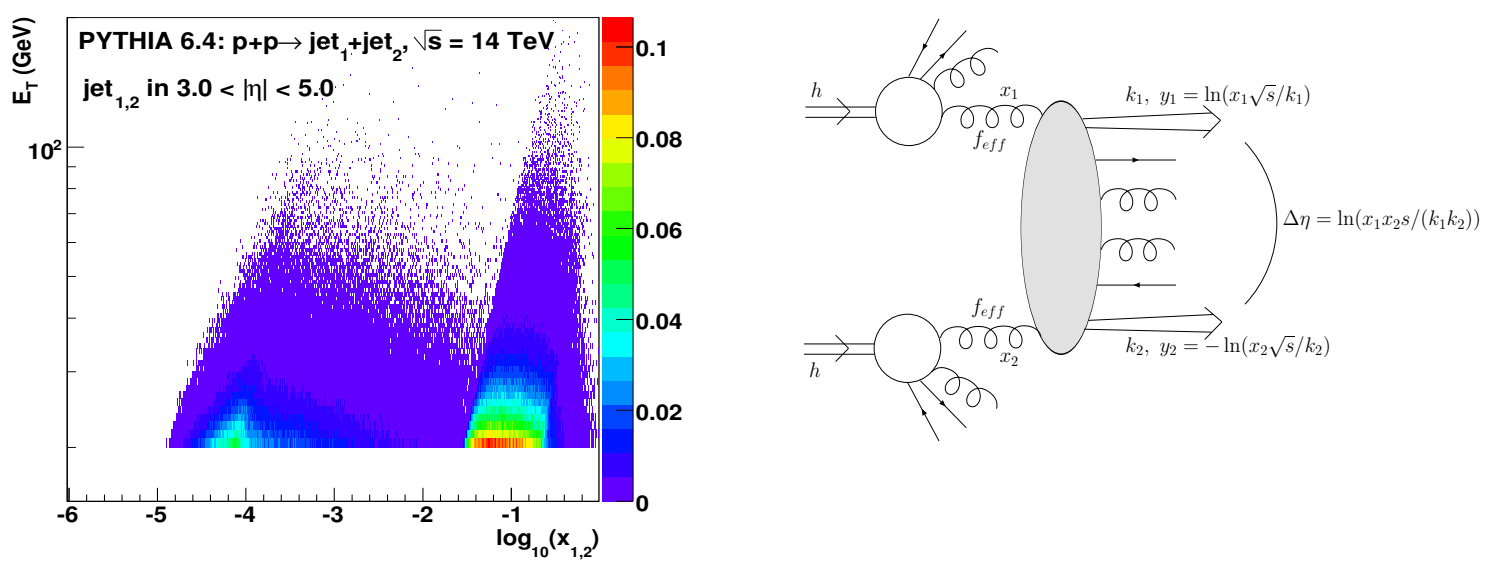

Figure 8: Left: Parton $x_{1,2}$ distributions probed in p-p collisions at $\sqrt{s}=14 \mathrm{TeV}$ with single jet production within ATLAS/CMS forward calorimeters acceptances. Right: MüllerNavelet dijet production diagram in p-p collisions.

- Case study II: Forward heavy-quarks

The possibility of ALICE and LHCb (Fig. 9, left) to reconstruct heavy D and B mesons as well as quarkonia in a large forward rapidity range can also put stringent constraints on the gluon structure and evolution at low- $x$. Studies of small- $x$ effects on heavy flavour production based on collinear and $k_{T}$ factorization, including non-linear terms in the parton evolution, lead to varying predictions for the measured $c$ and $b$ cross-sections at the LHC [37]. The hadroproduction of $J / \psi$ proceeds mainly via gluon-gluon fusion and, having a mass around the saturation scale $Q_{s}^{\mathrm{LHC}} \approx 3 \mathrm{GeV}$, is also a sensitive probe of possible gluon saturation phenomena. Figure 9 right, shows the gluon $x$ range probed in p-p collisions producing a $J / \psi$ inside the ALICE muon arm acceptance $(2.5 \lesssim \eta \lesssim 4)$. The observed differences in 
the underlying PDF fits translate into variations as large as a factors of $\sim 2$ in the finally measured cross sections [38].
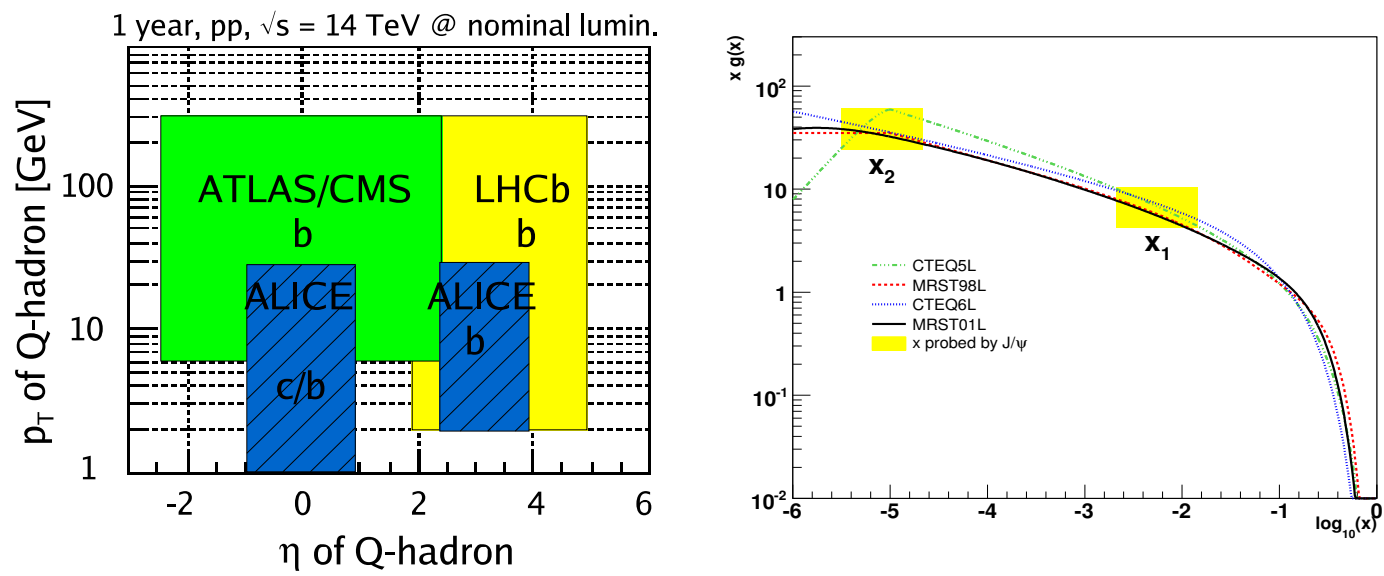

Figure 9: Left: Acceptances in $\left(\eta, p_{T}\right)$ for open charm and bottom at the LHC [37]. Right: Sensitivity of the forward $J / \psi$ measurement in ALICE to the gluon PDF [38].

\section{- Case study III: $\bar{Q} \bar{Q}$ exclusive photoproduction}

Ultra-peripheral interactions (UPCs) of high-energy heavy ions generate strong electromagnetic fields which can be used to constrain the low- $x$ behaviour of the nuclear gluon density via exclusive photoproduction of quarkonia, dijets and other hard processes [39]. Lead beams at $2.75 \mathrm{TeV}$ have Lorentz factors $\gamma=2930$ leading to maximum (equivalent) photon energies $\omega_{\max } \approx \gamma / R \sim 100 \mathrm{GeV}$, and corresponding maximum c.m. energies: $W_{\gamma \gamma}^{\max } \approx 160 \mathrm{GeV}$ and $W_{\gamma-\mathrm{A}}^{\max } \approx 1 \mathrm{TeV}$, i.e. $3-4$ times higher than equivalent photoproduction studies at HERA. The $x$ values probed in $\gamma \mathrm{A} \rightarrow \mathrm{Q} \overline{\mathrm{Q}}$ A processes (Fig. 10, left) can be as low as $x \sim 10^{-5}$ [39]. The ALICE, ATLAS and CMS experiments can measure the $J / \psi, \Upsilon \rightarrow e^{+} e^{-}, \mu^{+} \mu^{-}$produced in electromagnetic $\mathrm{Pb}-\mathrm{Pb}$ collisions tagged with neutrons detected in the ZDCs, as done at RHIC [40]. Full simulation+reconstruction analyses [41] indicate that CMS can measure a total yield of $\sim 500$ within $|\eta|<2.5$ for $0.5 \mathrm{nb}^{-1}$ nominal $\mathrm{Pb}-\mathrm{Pb}$ integrated luminosity (Fig. 10). Which such statistics, studies of the $p_{T}$ and $\eta$ distributions of the $\Upsilon$ can be carried out, helping to constrain the low- $x$ gluon density in the $\mathrm{Pb}$ nucleus.

- Case study IV: Forward Drell-Yan pairs

High-mass Drell-Yan pair production at the very forward rapidities covered by LHCb and by the CASTOR and TOTEM T2 detectors can probe the parton densities down to $x \sim 10^{-6}$ at much higher virtualities $M^{2}$ than those accessible in other measurements discussed here. A study is currently underway in CMS [3] to combine the CASTOR electromagnetic energy measurement together with the good position resolution of $\mathrm{T} 2$ for charged tracks, to trigger on and reconstruct $e^{+} e^{-}$pairs in p-p collisions at $14 \mathrm{TeV}$, and scrutinise $x g$ in the $M^{2}$ and $x$ plane. 

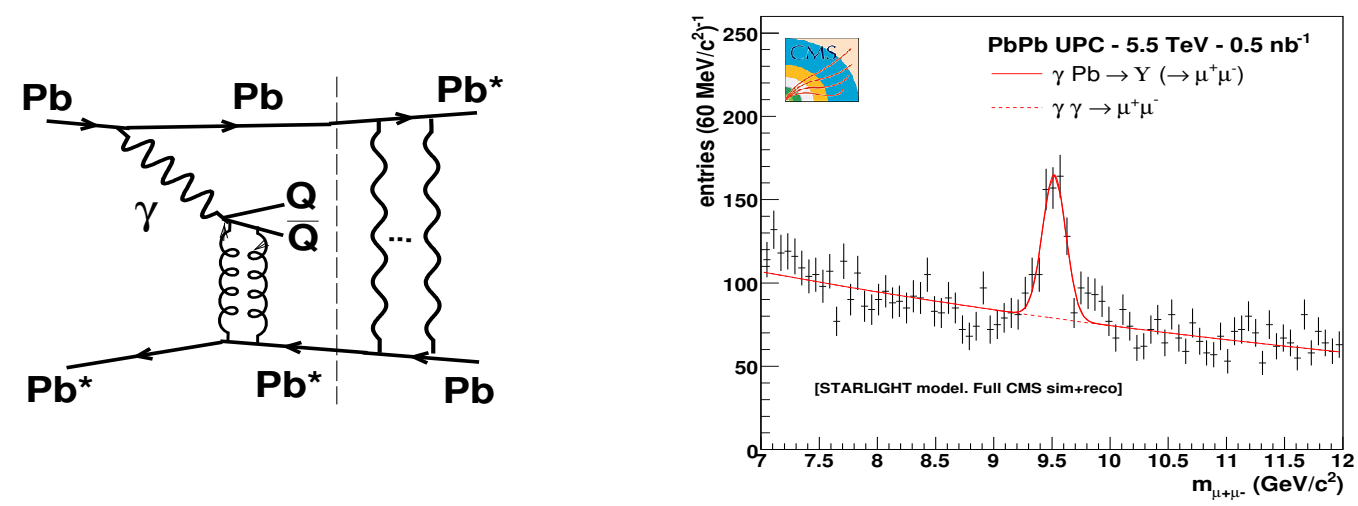

Figure 10: Left: Exclusive quarkonia photoproduction in UPCs. Right: Expected dimuon invariant mass from $\gamma \mathrm{Pb} \rightarrow \Upsilon \mathrm{Pb}^{\star}$ on top of $\gamma \gamma \rightarrow \mu^{+} \mu^{-}$continuum in UPC Pb-Pb at 5.5 $\mathrm{TeV}[41]$.

\section{Cosmic-rays physics connection}

The origin of cosmic rays (CRs) with energies above $10^{15} \mathrm{eV}$ is unclear, as it is the identity of the primaries. Due to their low fluxes (less than 1 particle per $\mathrm{m}^{2}$ and year) only indirect measurements exist which use the atmosphere as a "calorimeter" (Fig. 11, left). The energy and mass of UHE cosmic rays are then obtained with the help of Monte Carlo codes which describe the shower development (dominated by forward and soft QCD interactions) in the upper atmosphere [22]. The existing MC models (Fig. 11, right) predict energy and multiplicity flows differing by factors as large as three, with significant inconsistencies in the forward region $(|\eta|>5)$. Forward measurements at LHC energies $\left(E_{l a b} \approx 10^{17} \mathrm{eV}\right)$ in p-p, p$\mathrm{A}$ and $\mathrm{A}-\mathrm{A}$ collisions ${ }^{\mathrm{b}}$ will provide strong constraints to calibrate and tune these models and make more reliable predictions for the CR energy and composition at the highest energies observed. Forward measurements at the LHC, especially in calorimeters with longitudinal segmentation like CASTOR, will in addition help to interpret exotic CR topologies like the so-called "Centauro" events [8].

\section{Electroweak physics}

Interesting electroweak processes in photon-photon and photon-proton,-nucleus interactions, tagged with forward instrumentation, will be also accessible for the first time at $\mathrm{TeV}$ energies at the LHC. Two-photon dilepton production, $\mathrm{pp} \rightarrow \mathrm{p} l^{+} l^{-} \mathrm{p}$ (Fig. 12, left) will be an excellent luminosity calibration process, with a very well known QED cross-section [42]. Experimentally, such a process can be tagged with forward protons and has a clear signature in the exclusive back-to-back dielectrons (dimuons) measured e.g. in CASTOR/T2 (in the central muon chambers). The p-p cross section calculated using LPAIR for events where both muons have $p_{T}>3 \mathrm{GeV} / c$ and can, therefore, reach the CMS muon chambers is about $50 \mathrm{pb}$. About 300 events per $100 \mathrm{pb}^{-1}$ are thus expected in CMS after muon trigger cuts [3]. The situation is much more favourable in the case of $\mathrm{Pb}-\mathrm{Pb}$ collisions since the

${ }^{\mathrm{b}}$ Note that CRs interactions in the atmosphere are mostly $\mathrm{p}, \alpha, \mathrm{Fe}-\mathrm{Air}$ collisions. 

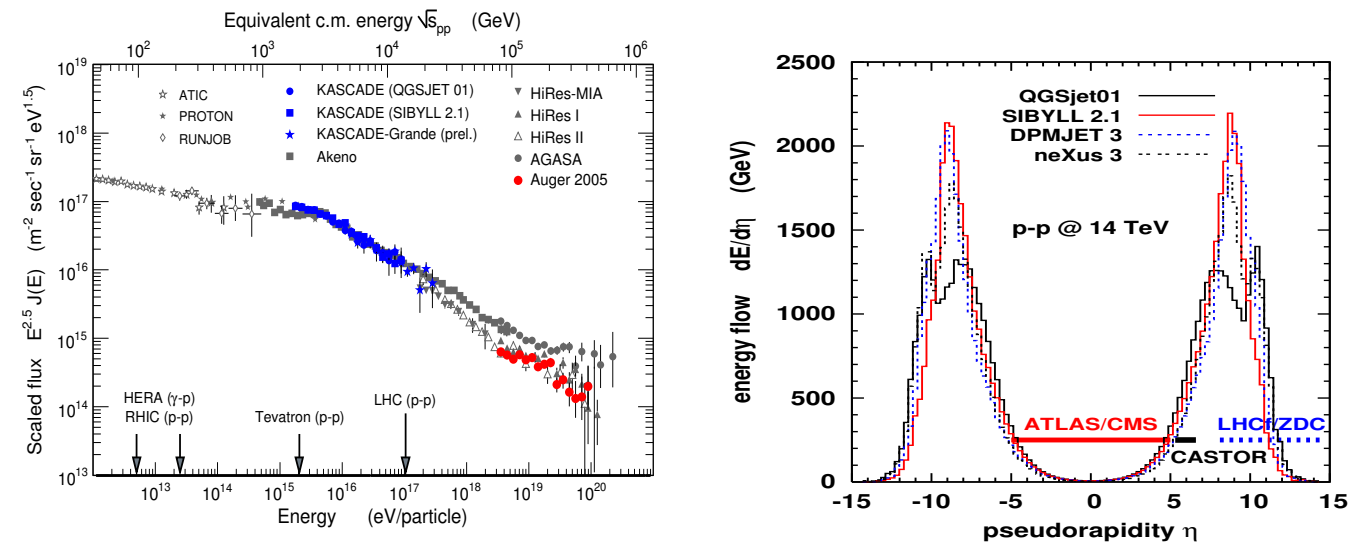

Figure 11: Left: The cosmic-ray energy distribution [22]. Right: Pseudo-rapidity energy distribution for p-p at the LHC predicted by four commonly used MC models in UHE cosmic rays physics (the acceptance of LHCf and ZDCs refers to neutral particles) [3].

dilepton continuum is much larger than in p-p ( $Z^{4}$ enhancement factor, see Fig. 10 right) and the forward neutron tagging much more efficient than the forward proton one.

The couplings of gauge bosons among themselves belong to one of the least tested sectors of the electroweak theory. A process well-suited to testing the $(W W \gamma)$ gauge boson selfinteraction is the photoproduction of single $W$ bosons from a nucleon (Fig. 12, right) in ultra-peripheral p-p [43], p-A and A-A [23] collisions. A large cross-section of about $1 \mathrm{pb}$ is expected for large photon-proton c.m. energies, $W_{\gamma p}>1 \mathrm{TeV}$. In addition, the two-photon $W^{+} W^{-}$exclusive production probes quartic gauge-boson-couplings. The process has a total cross-section of more than $100 \mathrm{fb}$, and a very clear signature. Its cross-section is still about $10 \mathrm{fb}$ for $W_{\gamma p}>1 \mathrm{TeV}$ showing sensitivity to physics beyond the SM [43].
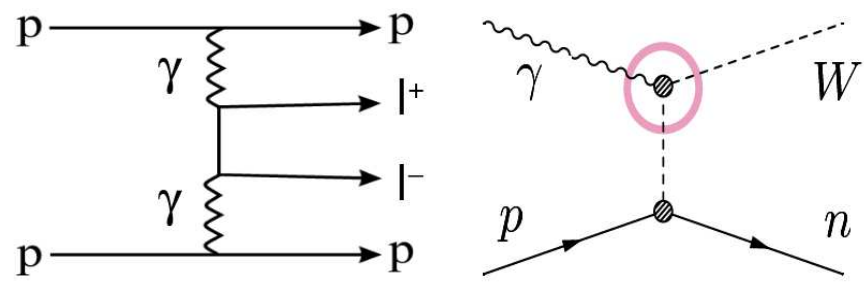

Figure 12: Photoproduction diagrams in electromagnetic proton-proton interactions: twophoton dilepton production (left), and single-W photoproduction (right). In both processes, the forward-going protons (neutrons) can be detected in RPs (ZDCs). 


\section{Acknowledgments}

I would like to thank the organisers of DIS'07 - and, in particular, the conveners of the Future of DIS Section - for their kind invitation to such a stimulating conference. Special thanks due to Albert de Roeck, Michele Arneodo and Monika Grothe for valuable discussions. Work supported by the 6th EU Framework Programme contract MEIF-CT-2005-025073.

\section{References}

[1] Slides: http://indico .cern. ch/contributionDisplay $\cdot$ py? contribId=248\&sessionId=10\&conf Id=9499

[2] S. Roesler, R. Engel and J. Ranft, arXiv:hep-ph/0012252 (2000).

[3] M. Albrow et al. [CMS/TOTEM Collabs.], Prospects for diffractive and forward physics at the LHC, CERN-LHCC-2006-039 (2006).

[4] ATLAS: Detector and Physics performance TDR, CERN/LHCC-99-14 (1999).

[5] S. Ask [ATLAS Collaboration], these Proceedings, arXiv:0706.0644 [hep-ex] (2007).

[6] CMS Physics TDR, Volume I: Detector Performance and Software, CERN/LHCC-2006-001 (2006).

[7] K. Borras [CMS Collaboration], these Proceedings

[8] A. L. S. Angelis et al., Nuovo Cim. 24C 755 (2001).

[9] O. A. Grachov et al., AIP Conf. Proc. 867 (2006).

[10] F. Carminati et al. [ALICE Collaboration], J. Phys. G 30, 1517 (2004).

[11] LHCb, Reoptimised Detector Design and Performance TDR, CERN/LHCC 2003-030, (2003).

[12] ALICE TDR: Zero degree calorimeter (ZDC), CERN-LHCC-99-05 (1999).

[13] V. Berardi et al. [TOTEM Collaboration], CERN/LHCC-2004-002 (2004).

[14] Technical Design Report of the LHCf experiment, CERN-LHCC-2006-004 (2006).

[15] M. G. Albrow et al., FP420 R\&D proposal, CERN/LHCC-2005-025 (2005).

[16] A. Pilkington [FP420 Collaboration], these Proceedings

[17] M. Arneodo and M. Diehl, arXiv:hep-ph/0511047 (2005).

[18] K. Goulianos, arXiv:0707.1055 [hep-ex] (2007).

[19] A. De Roeck et al., Eur. Phys. J. C 25391 (2002).

[20] V. A. Khoze, A. D. Martin and M. G. Ryskin, arXiv:0705.2314 [hep-ph], and refs. therein (2007).

[21] D. d'Enterria, Eur. Phys. J. A 31, 816 (2007).

[22] R. Engel, Nucl. Phys. Proc. Suppl. 151437 (2006).

[23] A. Baltz et al., arXiv:hep-ph/0702212 (2007).

[24] C. Bourrely, N. N. Khuri, A. Martin, J. Soffer and T. T. Wu, arXiv:hep-ph/0511135 (2005).

[25] J. R. Cudell et al. [COMPETE Collaboration], Phys. Rev. Lett. 89201801 (2002).

[26] G. Anelli et al. [TOTEM Collaboration], arXiv:hep-ex/0602025 (2006).

[27] M. E. Convery [CDF Collaboration], Acta Phys. Polon. B 36665 (2005).

[28] T. Teubner, these Proceedings.

[29] M. Klein, these Proceedings.

[30] N. Armesto, J. Phys. G32, R367 (2006)

[31] R. Venugopalan, these Proceedings.

[32] See http://durpdg.dur.ac.uk/hepdata/pdf3.html

[33] A. H. Müller and H. Navelet, Nucl. Phys. B 282, 727 (1987)

[34] C. Marquet and C. Royon, Nucl. Phys. B 739, 131 (2006)

[35] C. Royon, these Proceedings, arXiv:0706.1799 [hep-ph] (2007).

[36] A. S. Vera and F. Schwennsen, these Proceedings, arXiv:0707.0256 [hep-ph] (2007).

[37] J. Baines et al., Proceeds. HERA-LHC Workshop, hep-ph/0601164 (2006).

[38] D. Stocco [ALICE Collaboration], High energy QCD ECT* Workshop, Trento (2007).

[39] K. Hencken et al., J. Phys. G to appear, arXiv:0706.3356 (2007).

[40] D. d'Enterria, Proceeds. Quark Matter'05, nucl-ex/0601001 (2006). 
[41] D. d'Enterria (ed.) et al., CMS Physics TDR: High Density QCD with Heavy-Ions, J. Phys. G to appear.

[42] D. Bocian and K. Piotrzkowski, Acta Phys. Polon. B 352417 (2004).

[43] S. Ovyn et al., Proceeds. Photon'07 Int. Conf., (2007). 